\title{
Survival Time of Adult Ischemic Stroke Patients and Associated Risk Factors: A Retrospective Cohort Study at FelegeHliwot Referral Hospital
}

\author{
Abay Kassie ${ }^{1}$, Salie Ayalew ${ }^{2}$, Mandefro Abere ${ }^{2}$ \\ ${ }^{1}$ Department of Statistics, College of Natural and Computational Sciences, Debarq University, Gondar, Ethiopia, ${ }^{2}$ Department of Statistics, College of Natural and \\ Computational Sciences, University of Gondar, Gondar, Ethiopia.
}

\section{Abstract}

Background: Stroke is a major public health and clinical problem that represents the third leading cause of disease worldwide among adult non-communicable diseases. Stroke in the developing world is becoming a leading cause of death from infectious diseases that affects mainly adults and adult disability. Ischemic stroke is the most common form of strokes approximately about $80 \%-85 \%$ of all strokes in nature. Subjects and Methods: A retrospective cohort study was conducted at the Felege-Hiwot Referral Hospital among 232 ischemic stroke patients who started treatment between September 2014 and August 2016. Information on relevant variables was collected from adult ischemic stroke patients paper based medical cards and registries. The Kaplan-Meier survival curves and log-rank test used to compare the survival time of different category of predictors, and Cox's regression model employed to identify the effect of covariates on the survival time of ischemic stroke patients. Results: A total of 232 adult ischemic stroke, patients were included in the study. Out of the total 232 individuals, 69(29.74\%) died and the remaining 163(70.26\%) were censored at the end of follow-up period. Based on Cox proportional hazards regression model, age $(\mathrm{AHR}=1.015, \mathrm{CI}: 1.001-1.0304)$, diabetes $(\mathrm{AHR}=0.353, \mathrm{CI}$ : 0.1938- 0.643), hypertension $(\mathrm{AHR}=0.607$, 95\% CI:1.003-1.0053), structural heart disease (AHR $=2.231,95 \%$ CI: $1.158-4.593$ ), and history of previous ischemic stroke(AHR=1.84, 95\% CI: 1.088 - 3.113) were significantly associated with the mortality of adult ischemic stroke patients. Conclusion: Based on the Cox Proportional Hazards regression model the following prognostic factors, age, diabetes, hypertension, structural heart disease, fibrillation, and history of previous ischemic stroke were the risk factors for survival time of ischemic stroke patients.

Keywords: Cox Proportional Hazard Models, Ischemic Stroke, and Survival Function.

Corresponding Author: Mandefro Abere, Department of Statistics, College of Natural and Computational Sciences, University of Gondar, Gondar, Ethiopia

Received: December 2019

Accepted: December 2019

\section{Introduction}

Stroke is a major public health and clinical problem that represents the third leading cause of disease worldwide among adult non-communicable diseases, worldwide stroke was estimated to be about with approximately 16 million people developed first-ever-strokes and 62 million stroke survivors. Also stroke causes about 5.5 million deaths annually in their lifetime, from this stroke disease, which accounts for more than 85 percent of all cases of Ischemic stroke patients. ${ }^{[1]}$

According to world health organization (WHO), much of the burden of deaths resulting from stroke, estimated to be $10.8 \%$ mortality and $3.1 \%$ of the disease burden in worldwide, this can be attributed to patients are present late due to the standard of care is lower compared to hospitals in developed countries. ${ }^{[2]}$ Ischemic stroke in young adults still remains a significant health problem on individuals, their families, and societies due to ongoing demographic changes, including ageing of the population and health transitions observed in developing countries. In young adults, it accounts for approximately $10 \%-30 \%$ of all stroke patients in India and 3\%-8.5\% in Western countries. ${ }^{[3-6]}$

In Sub Saharan countries shows, there is an increasing clinical and epidemiological trend of stroke. However, good quality organized data on the prevention and treatment of stroke in Sub Saharan is still deficient and insufficient. Similarly, Ethiopia is also not different like sub Saharan countries, there is no well-organized and documented data. Reports in 2015, from the university of Gondar referral hospital showed a low prevalence of stroke (15 per $100,000)$ in a rural community based study in Ethiopia during 1986-1989. ${ }^{\text {[7-12] }}$

In South Africa (SA), the burden of stroke is responsible for accounts 25,000 deaths annually and 95,000 years lived with disability. According to the current evidence there is no prospective trial in the treatment and control of ischemic stroke in national vital registration from Ethiopia, is similar to that in the other limited publication of treatment has been done in South Africa. ${ }^{[8-10]}$

In Sub-Saharan Africa, most studies of stroke are hospital based case series. Hospital-based incidences are lower than in high-income regions, but higher in young people, possibly due to hospital admission bias. There are no community-based data on case fatality, but hospital-based 
case fatality is higher than elsewhere. The prevalence of stroke is lower than in high-income regions, but disabling stroke is just as prevalent. ${ }^{[13]}$

Globally, stroke is health problem of the serious leading cause of disability and the leading cause of mortality worldwide that frequently reduces the level of quality of life (QOL) of the survivors. Most risk factors have shown to influence the quality of life of those patients, approximately $5-8 \%$ of all ischemic strokes present in individuals younger than 45 years and above the age of 45 years, the occurrence of stroke begins to increase exponentially. Closely one quarter of ischemic strokes caused by non-conventional or unknown causes. In developing countries like Sub-Saharan Africa, the proportion of young ischemic stroke disability is higher and the overall mortality is high in the Western World. ${ }^{[14-16]}$

The causes of ischemic stroke are numerous and multifaceted, it occurs because of the supply of blood and oxygen to the brain is blocked. This accident emergency occurs due to blood clots in an artery to the brain or a narrowing of the arteries blocking. The blockage results, there is no enough amount of blood being reached in a timely to that portion of the brain. In addition, ischemic stroke disease is the leading cause of disability in adults and each year millions of stroke survivors has to adapt to a life with restrictions in activities of daily living because of ischemic stroke disease. To surviving patients will often needs effective treatment, prevention and control of ischemic stroke, understanding of their influences on the overall disease burden, and adequate information on the distribution of risk factors will needed. ${ }^{[17]}$

Stroke in the developing world is becoming a leading cause of death from infectious diseases that affects mainly adults and adult disability. Globally, $80 \%$ of stroke deaths occur in low- and middle-income regions. Africa may be increasingly affected by the high burden of ischemic stroke due to the health transitions in line with changing their social, economic and demographic patterns there is no a clear documented data. ${ }^{[18]}$

Ischemic stroke is the most common form of strokes is approximately about $80 \%-85 \%$ of all strokes in nature. Stroke lead to disability in a long time unless addressed in a timely manner, and those patients usually require more excessive investigations in order to find an underlying cause of patients. It is one of the most important life-threatening and serious medical emergencies, which requires timely medical assessment, diagnosis and treatment. ${ }^{[19]}$

In Ethiopia, an ischemic stroke is becoming an increasingly serious public health issue and there are no complete or reliable records of data specific to the Ethiopian setting are limiting the formulation of an appropriate response. There are no precise data and no adequate study on the morbidity and mortality of ischemic stroke in Ethiopia, and also there is no a precise prevalence of ischemic stroke risk factors, like hypertension, structural heart disease, old age and diabetes that can significantly contribute to the prevalence of adult ischemic stroke cases in Ethiopia. Recently in Ethiopia, ischemic stroke patients are common. Yet, there is no study conducted about ischemic stroke in Ethiopia, particularly about associated risk factors, clinical presentation and outcomes have not been further investigated. ${ }^{[18]}$

Therefore, this study aimed to determine the risk factors of the death of ischemic Stroke, in a hospital and outcome of CT scan proven ischemic stroke patients admitted to FelegeHiwot referral Hospital. In addition, little research has been done on stroke patients, but not on ischemic stroke patients in Ethiopia. Moreover, this study explores the major risk factors of adult ischemic stroke patients using survival analysis, semi-parametric proportional hazards model (Cox model) of ischemic stroke patients at the FelegeHiwot referral Hospital.

\section{Subjects and Methods}

\section{Study Design and Study Area}

A retrospective cohort study was conducted at the FelegeHiwot Referral Hospital, Bahir Dar. Which is located in the regional state of Amhara, which is $560 \mathrm{Km}$ away from the capital city of Addis Ababa, Ethiopia.

\section{Study population and Study period}

The target population for this study were all patients' aged $\geq 18$ years with ischemic stroke at the FelegeHiwot Referral Hospital ischemic stroke clinic. Adult ischemic stroke patients who started the treatment at the FelegeHiwot Referral Hospital from period between September 2014 and August 2016 were followed for a maximum of 36 months.

\section{Sample size and Data Collection Procedures}

In this retrospective cohort study, during the 36-month study period, 251 patients with ischemic stroke were admitted at the FelegeHiwot Referral hospital. Thus, the study included 232 adult ischemic stroke patients in the analysis, where the others were excluded from the study, which had key missing information like treatment history, laboratory results, and their charts.

Data was collected using standardized structured questionnaire and relevant data have taken from ischemic stroke follow-up charts. Professional nurses collected the data and more experienced nurses are included for supervision.

The study reviews patient's ischemic stroke charts, intake forms and follow up charts of ischemic stroke patients. Each patient has one medical file containing all ischemic stroke notes, which includes the patient intake forms and ischemic stroke care and follow-up card, prepared by the Federal ministry of health (FMOH) uniformly used by clinicians to early identify and document clinical and epidemiological variables. Thus, in this study secondary data, which was collected from the patient, follow up records. Based on this record of the patients, the variables which were important for the study were selected by using the patient's unique identification number or the laboratory code. This has done by communicating with the nurses and counselors to get the medical record and other important information for the study. 


\section{Quality assessment/ Model Adequacy}

The results of the Cox model were only valid as long as the assumptions of the model are correct. We have assessed the overall goodness of fit using R-square, graphs of Cox-Snell residuals, and then we have checked the proportionality assumption for each covariate included in the final model. The adequacy of the preliminary final model was checked for the validity of the proportional hazards assumptionusing tests based on the variables in the model with survival time and assess their significance using the Wald test. In addition, the plot of the scaled Schoenfeld residuals was used to provide any additional insight into any departure from proportionality. The STATA output showed for testing proportionality assumption; we have seen the Wald chisquare values and the corresponding $\mathrm{p}$-values for each covariate. Since the $\mathrm{p}$-values for each covariates were greater than 0.05 , the proportionality assumption was satisfied. The global fit test also shows that the test statistic was not significant which indicates that the proportional hazards assumption was not violated.

Another method of testing the proportionality assumption is by using the interactions of covariates with the logarithm of survival times were modeled together with the main effects. The Wald test was used to check the significance of the interaction terms at the 5\% level of significance. The result of the test indicates that none of the interaction terms are significant at the 5\% level and the result reveals nonsignificance of time-dependent covariates. On the other hand, there are no covariates, which show a trend/pattern with time. This indicates that the hazard ratio would be constant over the study time showing that there was no sufficient evidence to reject the null hypothesis. In addition, the assumption of proportionality was also assessed graphically by plotting the scaled Schoenfeld and martingale residuals of each covariate against log time. Thus, there was no violation of the proportional hazards assumption.

\section{Statistical Data Analysis}

After data collection, the response was coded and entered into the computer using EPI info data version 7 and the data was analyzed by using STATA software. Once we have collected time to event data, descriptive analysis for survival data is our first task to present numerical or graphical (using a survival curve) summaries of the survival times in a particular group. In general, a statistical analysis should begin with a thoughtful and thorough univariate description of the data. Survivor function and hazard function are the two functions of central interest in summarizing survival data.

The survival time of patients staying in hospital was followed for different period of observation with a minimum of 1 months and a maximum of 36 months. Next, we compare the categorical variables in the data summary among different factors are statistically significant or not with the help of Kaplan-Meier survival estimates and the log rank test.

Kaplan Meier analysis was used to study survival pattern, in which the probability of survival in each month after treatment initiation was calculated followed by calculation of cumulative probability of survival by the end of that particular month. The Kaplan-Meier estimator was used to estimate the survival time (time of censoring) of a patient and construct survival curves to compare the survival experience of a patient between different categorical variables.

The time of death was ascertained by calculating the difference between the date of initiation and date of outcome. The Kaplan Meier curve was drawn to arrive at the overall estimate of patients surviving at the end of each month. The difference in the survival patterns over time between different groups was studied using the log rank test. The Cox proportional hazards model was used for multivariate analysis to identify the risk factors for death. Cox proportional hazards regression model for examining the covariate effects on the hazard function.

\section{Results}

\section{Demographic Characteristics}

Of the total 232 adult ischemic stroke patients the mean age of ischemic stroke patients was 58.62 years. Of the total, 9 $(22.50 \%)$ were Not married, whereas $30(29.20 \%)$ and 27 $(34.18 \%)$ Married and (Divorced/Widowed) were dying. With regard to residence, about $26.72 \%$ of the patients were urban from the total of 116 urban ischemic stroke patients. Of the total study participants,69(29.74\%) of the patients died and the remaining $163(70.26 \%)$ of the patients were censored. Of the total 103 of adult male ischemic stroke patients, 40(38.83\%) died and out of 129 female $29(22.48 \%)$ adult ischemic stroke patients died. [Table 1]

\section{Clinical Characteristics}

Out of 180 with the presence of diabetic patients considered in the analysis 51(28.33\%) died. From the total of 147 hypertension patients, $23.81 \%$ of ischemic stroke patients died and the remaining $40 \%$ of no hypertension were died. Among the ischemic stroke patients, $22.39 \%$ and $39.83 \%$ of the patients who had normal blood cholesterol and high blood cholesterol status were died respectively.

Of the total,37.01\% of the ischemic stroke patients have Structural heart disease and $15.38 \%$ of the ischemic stroke patients have no Structural heart disease. The majority of $34.85 \%$ of ischemic stroke patients had a previous history of ischemic stroke [Table 2]

\section{Comparisons of survivorship function}

The estimate of overall Kaplan-Meier survival function reveals that most of the deaths occurred in the last months of ischemic stroke patients with increasing the survival probability.[Figure 1]

The Kaplan-Meier estimator survival curve indicates the estimate of survivor functions among different groups of covariates to make comparisons. The effect of sex and hypertension on the probability of death of the adult ischemic stroke plot suggests that the higher probability of death of adult ischemic stroke in hypertensive and male 
patient were higher than that of non-hypertensive and a female patient respectively. [Figure 2\&3].

\section{Cox Proportional Hazard Regression Model \\ Univariable Analysis}

In the univariate analysis using Log-rank test for categorical variables showed that the covariates sex, age, diabetes, hypertension, multi-collinearity of diabetes and hypertension, heart disease, fibrillation, previous ischemic stroke and high cholesterol had a significant difference in the survival experience among their categories. These variables were selected as significant factors for the death of ischemic stroke patients in the multivariable Cox regression model.[Table 3]

\section{Multivariable Cox Proportional Hazards Model}

We consider the preliminary model, which included all the predictors that had p-values less than 0.25 in the univariate analysis. Sex, age, diabetes, hypertension, multi-collinearity of diabetes and hypertension, structural heart disease, fibrillation, previous ischemic stroke and high cholesterol were included in Multivariable Cox Proportional Hazards Model. Multivariable Cox proportional hazards regression model using the response variable, namely survival time of ischemic stroke, with censoring indicator variable along with the covariates was analyzed. In the univariate analysis using the log-rank test for the categorical variables, and univariate Cox proportional hazard model for the continuous variables, age, diabetes, hypertension, multicollinearity of diabetes and hypertension, structural heart disease, fibrillation and previous ischemic stroke were included for the multivariable Cox proportional hazard model were significant at 0.05 significant levels. [Table 4] Age: among adult ischemic stroke patients a one unit increase in the age of patients the instantaneous risk of death was increased by 1.02 . This shows that an increase of one year in age of patients increases the hazard rate of death by $2 \%$.

Co-factors of diabetes and hypertension: among adult ischemic stroke patients, the instantaneous risk of death with co-factors of diabetes and hypertension are 1.8 times the instantaneous risk of death without co-factors of diabetes and hypertension, after keeping all other covariates at some constant level. This indicates that adult ischemic stroke patients with co-factors of diabetes and hypertension have $80 \%$ excess risk of instantaneous death than adult ischemic stroke patients without co-factors of diabetes and hypertension.

Structural heart disease: Among adult ischemic stroke patients, the instantaneous risk of death with Structural heart disease were 2.31 times the instantaneous risk of death not having a structural heart disease, after controlling all other covariates at some constant level.

Previous ischemic stroke: Of the adult ischemic stroke patients, the instantaneous risk of death for having a previous history of ischemic stroke were 1.84 times the instantaneous risk of death for without having a previous history of ischemic stroke, after keeping all other covariates at some constant level.[Table 4]

Table 1: Summary of some important socio-demographic characteristics of adult ischemic stroke patients at FelegeHiwot Referral Hospital

\begin{tabular}{|c|c|c|c|c|}
\hline \multirow{2}{*}{$\begin{array}{l}\text { Demographic and } \\
\text { Health Factors }\end{array}$} & \multicolumn{4}{|c|}{ Summary of the Number of Event and Censored values } \\
\hline & Category & Censored (\%) & Event/death (\%) & Total (\%) \\
\hline \multirow[t]{2}{*}{ Sex of patient } & 0 (male) & $63(61.17)$ & $40(38.83)$ & 103 \\
\hline & 1 (female) & $100(77.52)$ & $29(22.48)$ & 129 \\
\hline \multirow{3}{*}{ Marital status } & 0 (Not married) & $31(77.50)$ & $9(22.50)$ & 40 \\
\hline & 1(Married) & $80(70.80)$ & $33(29.20)$ & 113 \\
\hline & 2(Others(Divorced/Widowed)) & $52(65.82)$ & $27(34.18)$ & 79 \\
\hline \multirow[t]{2}{*}{ Residence of patient } & 0(Urban) & $85(73.28)$ & $31(26.72)$ & 116 \\
\hline & 1(Rural) & $78(67.24)$ & $38(32.76)$ & 116 \\
\hline
\end{tabular}

Table 2: Summary of some important clinical characteristics of adult ischemic stroke patients at FelegeHiwot Referral Hospital

\begin{tabular}{|c|c|c|c|c|c|}
\hline \multirow{2}{*}{ Clinical factors } & \multicolumn{5}{|c|}{ Status of censored and Event values } \\
\hline & Category & Censored & Death & Death/event percentage & Total \\
\hline Diabetes status of patient & Yes & 129 & 51 & 28.33 & 180 \\
\hline \multirow[t]{3}{*}{ High cholesterol(mg/dl) } & $<200$ & 52 & 15 & 22.39 & 67 \\
\hline & $\geq 200$ & 71 & 47 & 39.83 & 118 \\
\hline & Undetermined & 40 & 7 & 14.89 & 47 \\
\hline \multirow[t]{2}{*}{ Structural heart disease } & No & 66 & 12 & 15.38 & 78 \\
\hline & Yes & 97 & 57 & 37.01 & 154 \\
\hline \multirow[t]{2}{*}{ clinical history of Atrial fibrillation } & No & 70 & 36 & 33.96 & 106 \\
\hline & Yes & 93 & 33 & 26.19 & 126 \\
\hline \multirow[t]{2}{*}{ Previous ischemic stroke } & No & 77 & 23 & 23.00 & 100 \\
\hline & Yes & 86 & 46 & 34.85 & 132 \\
\hline
\end{tabular}


Table 3: Log-rank test for equality of survival time among the different groups of covariates for adult ischemic stroke patients in FelegeHiwot Referral Hospital.

\begin{tabular}{|l|l|l|l|}
\hline Covariates & Chi square & DF & P - value \\
\hline Sex & 6.08 & 1 & 0.0136 \\
\hline Marital status of patients & 2.24 & 2 & 0.3267 \\
\hline Residence of patients & 1.01 & 1 & 0.3144 \\
\hline Diabetes Mellitus of patient & 4.43 & 1 & 0.0353 \\
\hline Hypertension status of patient & 4.14 & 1 & 0.0418 \\
\hline Co-factors factors of Diabetes and hypertension of patients & 4.13 & 1 & 0.0422 \\
\hline High cholesterol(mg/dl) & 11.10 & 2 & 0.0039 \\
\hline Structural heart disease & 6.41 & 1 & 0.0114 \\
\hline Atrial fibrillation of patient & 8.26 & 1 & 0.0041 \\
\hline Previous ischemic stroke & 4.15 & 1 & 0.0417 \\
\hline
\end{tabular}

Table 4: Results of Cox proportional hazards regression model for survival of adult ischemic stroke patients under study treated at Felege-Hiwot Hospital, in Bahir-Dar.

\begin{tabular}{|c|c|c|c|c|c|c|c|c|}
\hline \multirow[t]{2}{*}{ Covariates } & \multirow[t]{2}{*}{ Df } & \multirow[t]{2}{*}{$\mathbf{B}$} & \multirow[t]{2}{*}{ SE } & \multirow[t]{2}{*}{ Wald } & \multirow[t]{2}{*}{ Sig. } & \multirow[t]{2}{*}{$\operatorname{Exp}(B)$} & \multicolumn{2}{|c|}{ 95.0\% CI for $\operatorname{Exp}(B)$} \\
\hline & & & & & & & Lower & Upper \\
\hline Sex (male) & 1 & -0.4694 & 0.2478 & -1.89 & 0.058 & 0.6254 & 0.3848 & 1.0165 \\
\hline Age & 1 & 0.0152 & 0.0075 & 2.02 & 0.043 & 1.0153 & 1.0005 & 1.0304 \\
\hline Diabetes mellitus(No) & 1 & -1.0415 & -0.306 & -3.41 & 0.001 & 0.3529 & 0.1938 & 0.6427 \\
\hline Hypertension ( $\mathrm{No})$ & 1 & -0.4995 & 0.2576 & -1.94 & 0.032 & 0.6068 & 1.003 & 1.0053 \\
\hline $\begin{array}{l}\text { multi-collinearity of diabetes and } \\
\text { hypertension (No) }\end{array}$ & 1 & 0.5893 & 0.2782 & 2.12 & 0.034 & 1.8027 & 1.0450 & 3.120 \\
\hline heart disease (No) & 1 & 0.8355 & 0.3516 & 2.38 & 0.017 & 2.3059 & 1.1576 & 4.5931 \\
\hline Fibrillation(No) & 1 & -0.9534 & 0.2541 & -3.75 & 0.000 & 0.3854 & 0.2342 & 0.6343 \\
\hline Previous ischemic stroke(No) & 1 & 0.6097 & 0.2683 & 2.27 & 0.023 & 1.8400 & 1.0876 & 3.1128 \\
\hline $\begin{array}{l}\text { High cholesterol(undetermined) } \\
\text { Cholesterol }<200 \\
\text { Cholesterol }>=200\end{array}$ & $\begin{array}{l}2 \\
1 \\
1\end{array}$ & $\begin{array}{l}-0.1923 \\
0.5305\end{array}$ & $\begin{array}{l}0.4876 \\
0.4343\end{array}$ & -0.391 .22 & $\begin{array}{l}0.693 \\
0.222\end{array}$ & $\begin{array}{l}0.8250 \\
1.6998\end{array}$ & $\begin{array}{l}0.3172 \\
0.7257\end{array}$ & $\begin{array}{c}2.1452 \\
3.9814\end{array}$ \\
\hline
\end{tabular}

The reference is shown in brackets

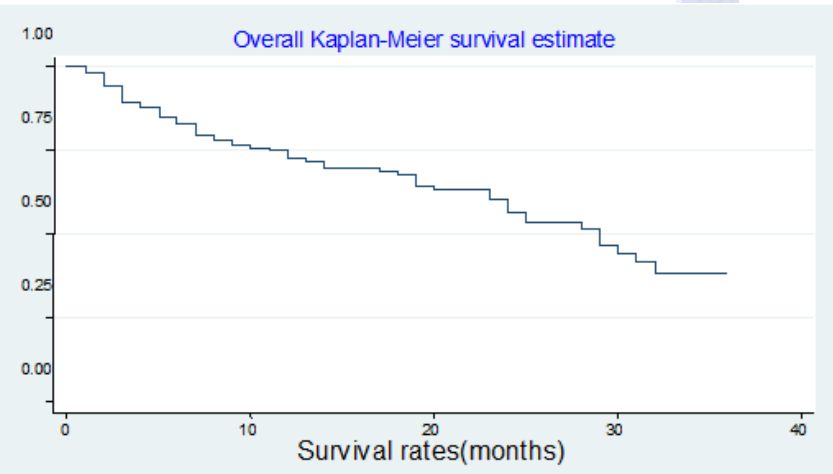

Figure 1: The plot of the overall estimate of Kaplan-Meier survivor function of adult ischemic stroke patients at FelegeHiwot Referral hospital.

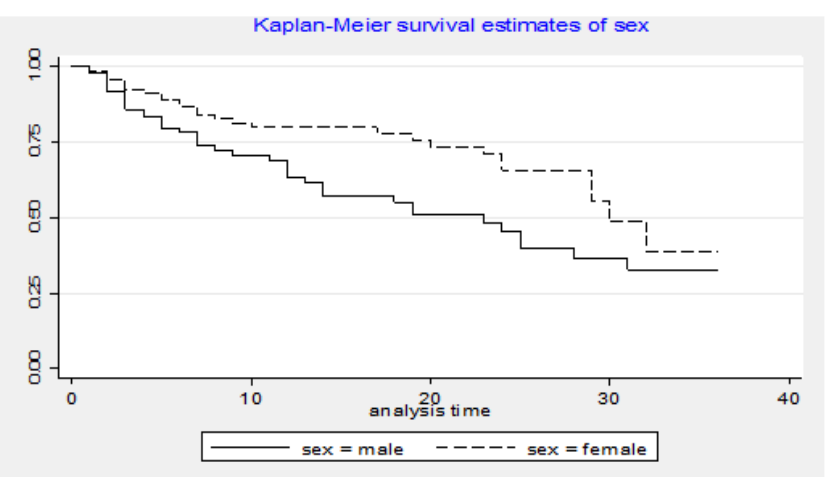

Figure 2: The plot of Kaplan-Meier survivor function, sex of adult ischemic stroke patients at FelegeHiwot Referral Hospital.

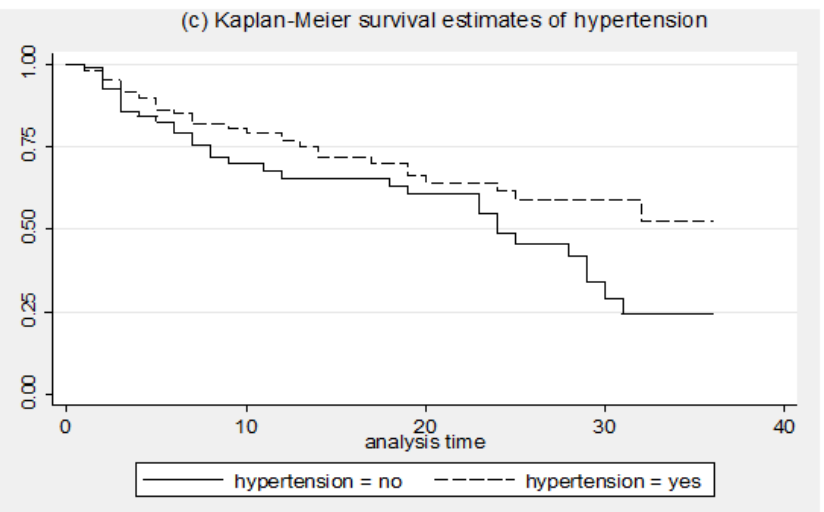

Figure 3: The plot of Kaplan-Meier survivor function, hypertension status of adult ischemic stroke patients at FelegeHiwot Referral Hospital.

\section{Discussion}

The survival probability was increased from early phase of ischemic stroke treatment follow-up to the end of the study. In this study, from the 103 male and 129 female adult ischemic stroke patients, $38.83 \%$ of males and $22.48 \%$ of females died and the mean age was 58.62 years. Similar study at Indraprastha Apollo Hospital, New Delhi on adult ischemic stroke patients had been reported 165 (67.6\%) were males, and $79(32.4 \%)$ were females from the total of 246 study subjects, also the mean age was $57.1 \pm 1.7$ years. ${ }^{[23]}$

Our study showed that age had a significant effect on 
survival time of adult ischemic stroke patients $(\mathrm{AHR}=1.015$, CI: $1.001-1.0304, \mathrm{p}=0.043)$. Thus, age was an important significant factor in survival time of adult ischemic stroke patients. A similar retrospective cohort study findings, by in Switzerland provided evidence that age was a strong risk factor for early death. ${ }^{[2]}$

A prospective longitudinal study conducted in Canada by. ${ }^{[25]}$ Shows that sex was the most significant risk factor of death during ischemic stroke treatment period. In our study, sex was not significantly associated with death of ischemic stroke patients.

The finding of this study shows that adult ischemic stroke patients with diabetes and hypertension had a higher risk of mortality than without diabetes and hypertension. After adjusting the other covariates, the hazard rate of adult ischemic stroke patients with diabetes was $65 \%$ less than the instantaneous death of ischemic stroke patients who have no diabetes (AHR=0.35, CI: 0.1938 - 0.6427).In addition, the hazard rate of ischemic stroke patients with a hypertension was $39 \%$ as lower than the instantaneous death of ischemic stroke patients who had no hypertension (AHR=0.610, CI: $1.003-1.0053$ ). This finding was in agreement with studies in Iran and South Indian showed that diabetes and hypertension was the most significant predictor of mortality for adult ischemic stroke patients. ${ }^{[26,27]}$

Structural heart disease and atrial fibrillation were other predictors of mortality in adult ischemic stroke patients. Especially, trial fibrillation is strongly associated with high mortality. This finding is supported by reports from other studies in Switzerland, ${ }^{[24]}$ and in Indraprastha Apollo. ${ }^{[23]}$ This suggests that atrial fibrillation was a significant risk factor of death during ischemic stroke treatment period. Our findings of the study using Cox proportional regression model shows that hypertension $(\mathrm{p}=0.032)$ and atrial fibrillation $(\mathrm{p}=0.000)$ were strongly significantly associated with death of adult ischemic stroke patients.

\section{Conclusion}

Based on the Cox Proportional Hazards regression model age, diabetes, hypertension, structural heart disease, fibrillation, and history of previous ischemic stroke were the risk factors of adult ischemic stroke patients. However, sex, residence and marital status were not associated with adult ischemic stroke patients.

\section{Limitation of the Study}

Since the study was based on secondary data, information on some important factors such as drinking alcohol, smoking status, were not available. Due to this, we have not used such variables in our models. As a result, our Cox proportional hazards regression model results may be biased.

\section{List of Abbreviations}

(AF) Atrial Fibrillation, (CVD) Cardiovascular Diseases, (CT) Computerized Tomography, (DALY) Disability
Adjusted Life Years, (DM)Diabetes Mellitus, (DOTS)Direct Observation Treatment Services, (DOTP)Direct Observation Treatment Program, (FMOH) Federal Ministry Of Health, (GBD) Global Burden of Disease, (HR) Hazard Ratio, (HPT) Hypertation, (ICU) Intensive Care Unit, (IS) Ischemic Stroke, (KM) KaplanMeier, (PIS) Previous Ischemic Stroke, (QOF) Quality of Life, (SSA) Sub Saharan Africa, (WHO) World Health Organization

\section{Declarations}

\section{Authors' contributions}

AK: The conception of research idea. SA, AK, and MA : analysis, interpretation and the drafting of the manuscript. All authors read and approved the final manuscript.

\section{Ethical consideration}

Ethical clearance was obtained from research and ethics committee of the Department of Statistics, University of Gondar.

\section{Competing interests}

The authors declare that they have no competing interests.

\section{Patients and public involvement}

Not applicable in this research

\section{Availability of data and materials}

Main part of the data generated or analyzed during this study were included in this published article. However, additional files of the research were also included in the journal.

\section{Consent for publication}

All participants provided written informed consent to publish this study.

\section{References}

1. Rutten-Jacobs LC, Arntz RM, Maaijwee NA, Schoonderwaldt HC, Dorresteijn LD, van Dijk EJ, et al. Long-term mortality after stroke among adults aged 18 to 50 years. Jama. 2013;309(11):1136-44.

2. Sanya EO, Desalu OO, Adepoju F, Aderibigbe SA, Shittu A, Olaosebikan O. Prevalence of stroke in three semi-urban communities in middle-belt region of Nigeria: a door to door survey. Pan African Medical Journal. 2015;20(1).

3. Bonita R, Mendis S, Truelsen T, Bogousslavsky J, Toole J, Yatsu F. The global stroke initiative. The Lancet Neurology. 2004;3(7):391.

4. Kaul S, Bandaru V, Suvarna A, Boddu DB. Stroke burden and risk factors in developing countries with special reference to India. Journal of the Indian Medical Association. 2009;107(6):358, 67-70.

5. Nencini P, Inzitari D, Baruffi M, Fratiglioni L, Gagliardi R, Benvenuti $\mathrm{L}$, et al. Incidence of stroke in young adults in Florence, Italy. Stroke. 1988;19(8):977-81.

6. Nagaraja D, Gururaj G, Girish N, Panda S, Roy A, Sarma G, et al. Feasibility study of stroke surveillance: data from Bangalore, India. 2009.

7. Tekle-Haimanot R, Abebe M, Gebre-Mariam A, Forsgren L, Heijbel J, Holmgren G, et al. Community-based study of neurological disorders in rural central Ethiopia. Neuroepidemiology. 1991;9(5):263-77.

8. Lester FT, Tsega E. The pattern of adult medical admissions in Addis Ababa, Ethiopia. East African medical journal. 1976;53(11):620.

9. Mamushet Y, Zenebe G, Addissie A. MEDICAL AND NEUROLOGICAL COMPLICATIONS AMONG STROKE PATIENTS ADMITTED FOR INPATIENT CARE IN ADDIS ABABA, ETHIOPIA. Ethiopian medical journal. 2015;53(1).

10. Abraham G, Abdulkadir J. Cerebrovascular accidents in Ethiopians. A review of 48 cases. East African medical journal. 1981;58(6):431-6.

11. Walker RW, Jusabani A, Aris E, Gray WK, Mitra D, Swai M. A 
prospective study of stroke sub-type from within an incident population in Tanzania. SAMJ: South African Medical Journal. 2011;101(5):338-44.

12. Connor MD, Modi G, Warlow CP. Accuracy of the Siriraj and Guy's Hospital stroke scores in urban South Africans. Stroke. 2007;38(1):628.

13. Connor MD, Walker R, Modi G, Warlow CP. Burden of stroke in black populations in sub-Saharan Africa. The Lancet Neurology. 2007;6(3):269-78.

14. Lloyd-Jones D, Adams R, Carnethon M, De Simone G, Ferguson TB, Flegal K, et al. Heart disease and stroke statistics-2009 update. Circulation. 2009;119(3):e21-e181.

15. Renna R, Pilato F, Profice P, Della Marca G, Broccolini A, Morosetti $\mathrm{R}$, et al. Risk factor and etiology analysis of ischemic stroke in young adult patients. Journal of stroke and cerebrovascular diseases. 2014;23(3):e221-e7.

16. Putaala J, Metso AJ, Metso TM, Konkola N, Kraemer Y, Haapaniemi $\mathrm{E}$, et al. Analysis of 1008 consecutive patients aged 15 to 49 with firstever ischemic stroke. Stroke. 2009;40(4):1195-203.

17. Ciccone A, Valvassori L, Nichelatti M, Sgoifo A, Ponzio M, Sterzi R, et al. Endovascular treatment for acute ischemic stroke. New England Journal of Medicine. 2013;368(10):904-13.

18. Lopez AD, Mathers CD, Ezzati M, Jamison DT, Murray CJ. Global and regional burden of disease and risk factors, 2001: systematic analysis of population health data. The Lancet. 2006;367(9524):174757.

19. Nurses AAoN. Guide to the care of the patient with ischemic stroke. AANN Reference Series for Clinical Practice Glenview, IL: American Association of Neuroscience Nurses. 2004.
20. Alemayehu B, Oli K. Stroke admission to Tikur Anbassa Teaching Hospital: with emphasis on stroke in the young. Ethiopian Journal of Health Development. 2002;16(3):309-15.

21. Gebremariam SA, Yang HS. Types, risk profiles, and outcomes of stroke patients in a tertiary teaching hospital in northern Ethiopia. eNeurologicalSci. 2016;3:41-7.

22. Greffie E, Mitiku T, Getahun S. Risk factors, clinical pattern and outcome of stroke in a referral hospital, Northwest Ethiopia. Clin Med Res. 2015;4(6):182-8.

23. Renjen P, Beg M, Ahmad K. Epidemiological study of incidence and risk factors of Ischemic stroke subtypes according to Trial of ORG 10172 in acute stroke treatment criteria: A 3 years, hospital-based study. International Journal of Medicine and Public Health. 2015;5(1):50-

24. Nedeltchev K, Renz N, Karameshev A, Haefeli T, Brekenfeld C, Meier N, et al. Predictors of early mortality after acute ischaemic stroke. Swiss medical weekly. 2010;140(17-18):254-9.

25. Llibre-Guerra J, Cepero AV, Concepción OF, Llibre-Guerra J, Gutiérrez R, Llibre-Rodriguez J. Stroke incidence and risk factors in Havana and Matanzas, Cuba. Neurología (English Edition). 2015;30(8):488-95.

26. Fahimfar N, Khalili D, Mohebi R, Azizi F, Hadaegh F. Risk factors for ischemic stroke; results from 9 years of follow-up in a population based cohort of Iran. BMC neurology. 2012;12(1):117.

27. Feigin VL, Krishnamurthi RV, Parmar P, Norrving B, Mensah GA, Bennett DA, et al. Update on the global burden of ischemic and hemorrhagic stroke in 1990-2013: the GBD 2013 study. Neuroepidemiology. 2015;45(3):161-76.

Copyright: (C) the author(s), 2019. It is an open-access article distributed under the terms of the Creative Commons Attribution License (CC BY 4.0), which permits authors to retain ownership of the copyright for their content, and allow anyone to download, reuse, reprint, modify, distribute and/or copy the content as long as the original authors and source are cited.

How to cite this article: Kassie A, Ayalew S, Abere M. Survival Time of Adult Ischemic Stroke Patients and Associated Risk Factors: A Retrospective Cohort Study at FelegeHiwot Referral Hospital. Asian J. Med. Res. 2019;8(4):ME29-ME35.

DOI: dx.doi.org/10.21276/ajmr.2019.8.4.ME5 